\title{
Remark on the anisotropic prescribed mean curvature equation on arbitrary domains
}

\author{
Thomas Marquardt
}

Received: 1 December 2008 / Accepted: 22 December 2008 / Published online: 22 January 2009

(C) The Author(s) 2009. This article is published with open access at Springerlink.com

\begin{abstract}
In this article we consider the Dirichlet problem for hypersurfaces of anisotropic prescribed mean curvature $H=H(x, u, N)$ depending on $x \in \Omega \subset \mathbb{R}^{n}$, the height $u$ of the hypersurface $M=\operatorname{graph} u$ over $\Omega$ and the unit normal $N$ to $M$ at $(x, u)$. We give a condition relating $H$ and the mean curvature of $\partial \Omega$ that guarantees the existence of smooth solutions even for not necessarily convex domains.
\end{abstract}

Keywords Dirichlet problem - Anisotropic prescribed mean curvature $\cdot$ Boundary gradient estimate

Mathematics Subject Classification (2000) $\quad 35 \mathrm{~J} 25 \cdot 53 \mathrm{~A} 10$

\section{Introduction}

Let $\Omega \subset \mathbb{R}^{n}$ be a bounded domain and $M$ a hypersurface over $\Omega$ given as the graph of $u: \Omega \rightarrow \mathbb{R}$. The Dirichlet problem of prescribed anisotropic mean curvature is given by

$$
\frac{1}{n} \operatorname{div}\left(\frac{D u}{\sqrt{1+|D u|^{2}}}\right)=H(x, u, N(D u)) \quad \text { in } \Omega, \quad u=\varphi \quad \text { on } \partial \Omega .
$$

The left-hand side of the first equation is the average of the principal curvatures of $M$, i.e. the mean curvature. $H=H(x, z, N)$ is a given function depending on the point $(x, u) \in M$ and the direction of the upper normal to $M$ at that point, given by

$$
N(D u)=\frac{1}{\sqrt{1+|D u|^{2}}}\left(\begin{array}{c}
-D u \\
1
\end{array}\right) .
$$

T. Marquardt $(\varangle)$

Max-Planck-Institut für Gravitationsphysik, Albert-Einstein-Institut,

Am Mühlenberg 1, 14476 Golm, Germany

e-mail: thomas.marquardt@aei.mpg.de 
Early results of Serrin [4] for $H=H(x)$ are summarised in [3], where existence was proved using a fixed point argument and Hölder estimates for the gradient, reducing the proof to $C^{1}$-a priori estimates.

In the general case $H=H(x, z, N)$ either the condition

$$
\int_{\Omega} \sup _{z \in \mathbb{R}, N \in S^{n}}|H(x, z, N)|^{n} \mathrm{~d} x<\omega_{n}
$$

or the assumption $D_{z} H \geq 0$ together with

$$
\left|\int_{\Omega} H(x, 0, N(D \eta)) \eta \mathrm{d} x\right| \leq \frac{1-\varepsilon}{n} \int_{\Omega}|D \eta| \mathrm{d} x \quad \forall \eta \in C_{0}^{1}(\Omega)
$$

for some $\varepsilon>0$ ensure a bound on $u$. The result is well known in the case that (1) holds and can be established by applying the method of Stampacchia [5] otherwise. A condition comparable to (2) was first imposed by Giaquinta [2].

To derive a gradient estimate it is favourable to impose for some $\beta>0$ the structure condition

$(s c)\left\{\begin{array}{l}H_{1}, H_{2} \in C^{1}\left(\bar{\Omega} \times \mathbb{R} \times \mathbb{R}^{n+1}\right) \cap C^{1, \beta}\left(\Omega \times \mathbb{R} \times \mathbb{R}^{n+1}\right), \\ H(x, z, N)=H_{1}(x, z, N)+H_{2}(x, z, N) N_{n+1}, \quad D_{z} H_{1} \geq 0 \text { on } \Omega \times \mathbb{R} \times S_{+}^{n} .\end{array}\right.$

This condition was first used by Bergner [1]. He estimated $D u$ in the interior in terms of the boundary gradient using a maximum principle. The boundary gradient estimate he obtained was only valid in convex domains.

The main result of this paper is Theorem 1, where we prove a boundary gradient estimate assuming an a priori bound for $u,(s c)$ and the Serrin type condition

$$
H_{\partial \Omega}(y) \geq \frac{n}{n-1}\left|H_{1}(y, \varphi(y), \gamma(y))\right| \quad \forall y \in \partial \Omega,
$$

where $\gamma$ is the inward pointing unit normal of $\partial \Omega$ considered as a vector in $\mathbb{R}^{n+1}$. As a consequence we obtain existence of smooth solutions in Corollary 1 even for not necessarily convex domains.

\section{Results}

For a domain $\Omega \subset \mathbb{R}^{n}$ with $C^{2}$-boundary we define the boundary strip

$$
\Gamma_{1}:=\left\{x \in \bar{\Omega} \mid \mathrm{d}(x):=\operatorname{dist}(x, \partial \Omega)<d_{1}\right\}
$$

with $d_{1} \leq 1$ such that $\mathrm{d} \in C^{2}\left(\bar{\Gamma}_{1}\right)$ (cf. [3, Lemma 14.16]). Using $a:=\sup _{\Gamma_{1}}|\varphi|, m:=$ $\sup _{\Omega}|u|$ and

$$
\begin{aligned}
& h_{i}:=\sup _{\Gamma_{1} \times[-m, m] \times S^{n}}\left|H_{i}(x, z, N)\right| \quad \text { for } i=1,2, \\
& h_{1, X}:=\sup _{\Gamma_{1} \times[-a, a] \times S^{n}}\left|D_{X} H_{1}(x, z, N)\right| \text { for } X=x, z, N \text {, }
\end{aligned}
$$

we obtain the following result: 
Theorem 1 (Boundary gradient estimate) Let $n \geq 2$. Let $\Omega \subset \mathbb{R}^{n}$ be a bounded domain with $C^{2}$-boundary. Suppose that $\varphi \in C^{2}(\bar{\Omega})$. Let $u \in C^{2, \alpha}(\bar{\Omega})$ be a solution of the Dirichlet problem of anisotropic prescribed mean curvature with an a priori bound $m$. If $H$ satisfies (sc) and (3), then we have

$$
\sup _{\partial \Omega}|D u| \leq \sup _{\partial \Omega}|D \varphi|+\frac{1}{d_{1}}\left(1+2\|\varphi\|_{C^{1}\left(\bar{\Gamma}_{1}\right)}\right) e^{(1+C)(a+m)}
$$

with $C=C\left(n, \Omega,\|\mathrm{d}\|_{C^{2}\left(\bar{\Gamma}_{1}\right)},\|\varphi\|_{C^{2}\left(\bar{\Gamma}_{1}\right)}, h_{1}, h_{2}, h_{1, x}, h_{1, z}, h_{1, N}\right)$.

Remark 1 In the case $H=H(x, z)$, condition (3) reduces to the Serrin condition which is known to be sharp.

Corollary 1 (Existence and Uniqueness) Let $n \geq 2$. Let $\Omega \subset \mathbb{R}^{n}$ be a bounded domain with $C^{2, \alpha}$-boundary and $\varphi \in C^{2, \alpha}(\bar{\Omega})$ for some $\alpha \in(0,1)$. Suppose that $H$ satisfies $(s c),(1)$ and (3). Then the Dirichlet problem for the equation of anisotropic prescribed mean curvature has a solution $u \in C^{2, \alpha}(\bar{\Omega})$. In the case $D_{z} H \geq 0$ condition (1) can be replaced by (2). Furthermore if $D_{z} H \geq 0$ the solution is unique.

Remark 2 To generalise the result of Corollary 1 to boundary values $\varphi \in C^{0}(\partial \Omega)$ one can approximate $\varphi$ uniformly by functions $\varphi_{m} \in C^{2, \alpha}(\bar{\Omega})$. Demanding $D_{z} H \geq 0$ and applying a comparison principle, the hereby obtained solutions $u_{m} \in C^{2, \alpha}(\bar{\Omega})$ converge uniformly to some function $u \in C^{0}(\bar{\Omega})$ with $u=\varphi$ on $\partial \Omega$. Interior gradient estimates which follow from [6], Theorem 1.1 (one can easily see that they even hold in the case $H_{1, z} \geq 0$ ), together with interior Hölder and Schauder estimates imply by Arzelà-Ascoli that $u \in C^{0}(\bar{\Omega}) \cap C^{2}(\Omega)$ and $Q u=0$.

Remark 3 Translating solutions $u(x, t)=u_{0}(x)+t$ of mean curvature flow have the property that $u_{0}$ satisfies our Dirichlet problem with $H_{1} \equiv 0$ and $H_{2} \equiv 1 / n$. Hence Theorem 1 yields a boundary gradient estimate for such solutions in any mean convex domain. The necessary bound on $u_{0}$ can be shown explicitly, such that neither (1) nor (2) is needed.

Proof of Corollary 1 The conditions on $\Omega, \varphi$ and $H$ allow us to apply [3], Theorem 13.8 which reduces the existence proof to the derivation of $C^{1}$-a priori bounds for solutions $u \in C^{2, \alpha}(\bar{\Omega})$. Condition (1) on $H$ together with [3], Theorem 10.5 yields the a priori estimate of $u$. In the case $D_{z} H \geq 0$ this estimate can be obtained from (2) using the method of Stampacchia [5]. The estimate of $\mathrm{Du}$ in the interior can be established using the structure condition $(s c)$ and a maximum principle for the gradient as in [1]. Finally the boundary gradient estimate follows from Theorem 1, (sc) and condition (3). Uniqueness is obtained from $D_{z} H \geq 0$ by applying a comparison principle.

Proof of Theorem 1 We will use the elliptic operator $Q v:=a^{i j}(D v) D_{i j} v+b(x, v, D v)$ with

$$
\begin{aligned}
a^{i j}(p) & :=\left(1+|p|^{2}\right) \delta_{i j}-p_{i} p_{j}, \\
b(x, z, p) & :=-n\left(1+|p|^{2}\right)\left(|p-D \varphi| H_{1}(x, z, N(p))+b_{0}(x, p)\right), \\
b_{0}(x, p) & :=\left(\left(1+|p|^{2}\right)^{1 / 2}-|p-D \varphi|\right) H_{1}(x, u(x), N(p))+H_{2}(x, u(x), N(p)) .
\end{aligned}
$$

Note that solutions $u$ of the prescribed mean curvature equation satisfy $Q u=0$ and that by $D_{z} H_{1} \geq 0, b(x, z, p)$ is decreasing in $z$. We will prove that for some function $\psi$, which will 
be determined at the end, $w^{ \pm}:=\varphi \pm \psi \circ \mathrm{d}$ are global upper and lower barriers for $u$ and $Q$ in $\Gamma_{1}$. Therefore we need to show that the following holds:

$$
w^{ \pm}=u \quad \text { on } \partial \Omega, \quad w^{-} \leq u \leq w^{+} \quad \text { on } \partial \Gamma_{1} \backslash \partial \Omega \quad \pm Q w^{ \pm}<0 \quad \text { in } \Gamma_{1} \backslash \partial \Omega .
$$

Assuming $\psi^{\prime}(\mathrm{d}) \geq 1, \psi^{\prime \prime}(\mathrm{d}) \leq 0$ and using [3], Lemma 14.17 we first get

$$
\begin{aligned}
\pm & a^{i j}\left(D w^{ \pm}\right) D_{i j} w^{ \pm} \\
\leq & -\psi^{\prime}(\mathrm{d})\left(1+\left|D w^{ \pm}\right|^{2}\right)(n-1) H_{\partial \Omega}(y)+n^{2} \sup _{\Gamma_{1}}|D \varphi|^{2} \sup _{\Gamma_{1}}\left|D^{2} \mathrm{~d}\right| \psi^{\prime}(\mathrm{d})^{2} \\
& \quad+\psi^{\prime \prime}(\mathrm{d})+3 n^{2}\left(1+\sup _{\Gamma_{1}}|D \varphi|\right)^{2} \sup _{\Gamma_{1}}\left|D^{2} \varphi\right| \psi^{\prime}(\mathrm{d})^{2} \\
= & -\psi^{\prime}(\mathrm{d})\left(1+\left|D w^{ \pm}\right|^{2}\right)(n-1) H_{\partial \Omega}(y)+c_{1} \psi^{\prime}(\mathrm{d})^{2}+\psi^{\prime \prime}(\mathrm{d}),
\end{aligned}
$$

where $y \in \partial \Omega, \mathrm{d}(x)=|x-y|$ and $H_{\partial \Omega}$ denotes the mean curvature of $\partial \Omega$. Using this estimate in combination with

$$
\begin{gathered}
n\left|b_{0}\left(x, D w^{ \pm}\right)\right|\left(1+\left|D w^{ \pm}\right|^{2}\right) \leq 2 n\left(h_{1}+h_{2}\right)\left(1+\sup _{\Gamma_{1}}|D \varphi|\right)^{3} \psi^{\prime}(\mathrm{d})^{2}=: c_{2} \psi^{\prime}(\mathrm{d})^{2}, \\
n \psi^{\prime}(\mathrm{d})\left(1+\left|D w^{ \pm}\right|^{2}\right) \leq 2 n\left(1+\sup _{\Gamma_{1}}|D \varphi|\right)^{2} \psi^{\prime}(\mathrm{d})^{3}=: c_{3} \psi^{\prime}(\mathrm{d})^{3}
\end{gathered}
$$

and the monotonicity of $H_{1}(x, z, N)$ in $z$ we obtain for $\psi(\mathrm{d}) \geq 0$ :

$$
\begin{aligned}
\pm Q\left(w^{ \pm}\right) \leq & -\psi^{\prime}(\mathrm{d})\left(1+\left|D w^{ \pm}\right|^{2}\right)(n-1) H_{\partial \Omega}(y)+c_{1} \psi^{\prime}(\mathrm{d})^{2}+\psi^{\prime \prime}(\mathrm{d}) \\
& \mp n\left(1+\left|D w^{ \pm}\right|^{2}\right)\left|D w^{ \pm}-D \varphi\right| H_{1}\left(x, w^{ \pm}, N\left(D w^{ \pm}\right)\right) \\
& \mp n\left(1+\left|D w^{ \pm}\right|^{2}\right) b_{0}\left(x, D w^{ \pm}\right) \\
\leq & -\psi^{\prime}(\mathrm{d})\left(1+\left|D w^{ \pm}\right|^{2}\right)\left[(n-1) H_{\partial \Omega}(y) \pm n H_{1}(y, \varphi(y), \mp \gamma(y))\right] \\
& +c_{3} \psi^{\prime}(\mathrm{d})^{3}\left|H_{1}(y, \varphi(y), \mp \gamma(y))-H_{1}\left(x, \varphi(x), N\left(D w^{ \pm}(x)\right)\right)\right| \\
& +\left(c_{1}+c_{2}\right) \psi^{\prime}(\mathrm{d})^{2}+\psi^{\prime \prime}(\mathrm{d}) .
\end{aligned}
$$

The first term can be estimated using (1). For the second term we get from the condition $\psi^{\prime}(\mathrm{d}) \geq 2\|\varphi\|_{C^{1}\left(\bar{\Gamma}_{1}\right)}$ and with the help of

$$
\left|N\left(D w^{ \pm}(x)\right) \pm \gamma(y)\right| \leq 4\left(1+\sup _{\Gamma_{1}}|D \varphi|\right) \psi^{\prime}(\mathrm{d}(x))^{-1}
$$

the estimate

$$
\begin{aligned}
& \left|H_{1}(y, \varphi(y), \mp \gamma(y))-H_{1}\left(x, \varphi(x), N\left(D w^{ \pm}(x)\right)\right)\right| \\
& \quad \leq c(\Omega)\left(h_{1, x}+h_{1, z}\|\varphi\|_{C^{1}\left(\bar{\Gamma}_{1}\right)}\right) \mathrm{d}(x)+4 c(\Omega) h_{1, N}\left(1+\|\varphi\|_{C^{1}\left(\bar{\Gamma}_{1}\right)}\right) \psi^{\prime}(\mathrm{d}(x))^{-1} \\
& \quad=: r \mathrm{~d}+s \psi^{\prime}(\mathrm{d})^{-1} .
\end{aligned}
$$

Therefore if $\psi^{\prime}(\mathrm{d}) \mathrm{d} \leq 1$ we have finally

$$
\pm Q\left(w^{ \pm}\right) \leq\left(c_{1}+c_{2}+c_{3}(r+s)\right) \psi^{\prime}(\mathrm{d})^{2}+\psi^{\prime \prime}(\mathrm{d})=: C \psi^{\prime}(\mathrm{d})^{2}+\psi^{\prime \prime}(\mathrm{d}) .
$$

Choosing

$$
\psi:[0, \infty) \rightarrow \mathbb{R}: x \mapsto \psi(x):=\frac{\ln (1+\lambda x)}{1+C}, \quad \lambda:=\frac{e^{(1+C)(a+m)}-1}{d_{0}}
$$


we see that $\psi$ satisfies $\psi(\mathrm{d}) \geq 0, \psi^{\prime}(\mathrm{d}) \mathrm{d} \leq 1, \psi^{\prime \prime}(\mathrm{d})<0$ and also $\psi^{\prime}(\mathrm{d}) \geq 1+2\|\varphi\|_{C^{1}\left(\bar{\Gamma}_{1}\right)}$ for

$$
d_{0}:=\frac{e^{(1+C)(a+m)}-1}{(1+C)\left(1+2\|\varphi\|_{C^{1}\left(\bar{\Gamma}_{0}\right)}\right) e^{(1+C)(a+m)}} d_{1} .
$$

From the definition of $\psi$ and the calculations above we see that $w^{ \pm}$satisfy (*). From (*) we can derive that the following estimate holds:

$$
\sup _{\partial \Omega}|D u| \leq \sup _{\partial \Omega}|D \varphi|+\psi^{\prime}(0) .
$$

Thus the desired result follows.

Acknowledgments The author wants to thank Prof. Dr. Gerhard Huisken for the fruitful conversations which lead to this result.

Open Access This article is distributed under the terms of the Creative Commons Attribution Noncommercial License which permits any noncommercial use, distribution, and reproduction in any medium, provided the original author(s) and source are credited.

\section{References}

1. Bergner, M.: The Dirichlet problem for graphs of prescribed anisotropic mean curvature in $\mathbb{R}^{n+1}$. Int. Math. J. Anal. Appl. 28, 149-166 (2008)

2. Giaquinta, M.: On the Dirichlet problem for surfaces of prescribed mean curvature. Manus. Math. 12, 73-86 (1974)

3. Gilbarg, D., Trudinger, N.S.: Elliptic Partial Differential Equations of Second Order. Springer, Berlin (2001)

4. Serrin, J.: Gradient estimates for solutions of nonlinear elliptic and parabolic equations. Contributions to Nonlinear Functional Analysis, pp. 565-601. Academic Press, New York (1971)

5. Stampacchia, G.: Equations elliptiques du second ordre à coefficients discontinus. Ann. Inst. Fourier 15, 189-258 (1965)

6. Wang, X.-J.: Interior gradient estimates for mean curvature equations. Math. Z. 228, 73-81 (1998) 